\title{
The Influence of Nursing Safety Management on the Quality of Hemopurification Nursing
}

Dan Wang

The First Affiliated Hospital of Jinan University, Guangzhou 510630, Guangdong Province, China

Abstract: Objective: To study the influence of nursing safety management on the quality of hemopurification nursing. Methods: Fifty-six patients who underwent hemopurification in our hospital from October 2019 to October 2020 were selected and randomly divided into research group $(n=28)$ and control group $(n=28)$, control group patients adopt routine nursing mode, research group patient take nursing safety management mode. Then compare the nursing quality score, the occurrence of adverse events and nursing satisfaction of patients in the two groups after different nursing modes. Results: The service attitude, doctor-patient relationship, medical environment and professional skill scores of the research group were significantly higher than those of the control group $(P<0.05)$, the incidence of adverse events in the research group was significantly lower than that of the control group $(P<0.05)$, and the satisfaction of the patients in the research group was significantly higher than that of the control group $(P<0.05)$. Conclusion: The application of nursing safety management in hemopurification can effectively improve the quality of nursing, reduce the occurrence of adverse events, and improve the patient's nursing satisfaction, the effect is significant and suitable for promotion.

Key words: Nursing safety management; Hemopurification; Citric acid; Quality of nursing

Publication date: March, 2021

Publication online: 31 March, 2021

*Corresponding author: Dan Wang, 13073042384 @163.com

Hemopurification is a technique that requires a high level of knowhow on the part of the nursing staff and requires a strict concept of sterility.Patients undergoing hemopurification are at high risk of infection, as catheters often prolapse, which makes nursing more difficult and stressful, leading to adverse nursing events such as catheter infection, equipment failure, Hemodialysis bleeding, and falls, which can lead to disputes between nurses and patients. Therefore, to strengthen the safety management in nursing can improve the quality of hemopurification nursing $^{[1]}$. In this paper, 56 cases of patients who underwent hemopurification in our hospital from October 2019 to October 2020 were selected as the research object to study the impact of nursing safety management on the quality of hemopurification nursing. The details are as follows.

\section{Materials and methods}

\subsection{General information}

Fifty-six patients who underwent hemopurification in our hospital from October 2019 to October 2020 were selected and randomly divided into research group $(n=28)$ and control group $(n=28)$. In the research group, there were 16 males and 12 females, ranging in age from 75 years old to 25 years old, with an average age of $(49.68 \pm 4.21)$ years. The number of citric acid treatments was 85 , and the duration of hemodialysis was 0.5 to 5 years, with an average of (3.84 \pm 1.15$)$ years. Among them, there were 7 cases of gastrointestinal hemorrhage, 5 cases of cerebral hemorrhage, 10 cases of renal failure, 4 cases after kidney transplantation, 2 cases after hematopoietic stem cell transplantation. However, 8 patients were changed to heparin-free treatment midway, including 1 patient with elevated bilirubin, 4 patients with lactic 
acid greater than 15,2 patients with alkalosis, and 1 patient with total calcium to ionic calcium ratio greater than 2.5. In the control group, there were 15 males and 13 females, the highest age was 74 years old, the lowest age was 26 years old, and the average age was $(49.53 \pm 4.15)$ years old. The number of citric acid treatments was 86 , and the duration of hemodialysis ranged from 1 year to 4.5 years, with an average of (3.69 \pm 1.21$)$ years. Among them, there were 6 cases of gastrointestinal hemorrhage, 4 cases of cerebral hemorrhage, 11 cases of renal failure, 5 cases after kidney transplantation, 2 cases after hematopoietic stem cell transplantation. However, 10 patients were treated without heparin midway, among which 2 patients had elevated bilirubin, 3 patients had lactic acid greater than 15,3 patients had alkalosis, and 2 patients had total calcium to ion calcium ratio greater than 2.5. There was no significant difference in general data between the two groups $(P>0.05)$, which could be compared ${ }^{[2]}$.

\subsection{Methods}

All patients underwent continuous veno-venous hemofiltration $(\mathrm{CVVH})$ treatment mode, using the machine of Fresenius, using the filter of hollow fiber diafilter, the model of Polyflux $140 \mathrm{H}$, and using the replacement fluid formula as follows: $3000 \mathrm{ml} \mathrm{NS}$, $750 \mathrm{ml}$ of sterilized injection water and $125 \mathrm{ml}$ of $5 \%$ sodium bicarbonate injection, which can be adjusted appropriately according to the blood gas analysis of the patient; $10 \% \mathrm{KCl} 10 \mathrm{ml}$ and $50 \% \mathrm{GS} 10 \mathrm{ml}$, which can be adjusted on the basis of the patient's electrolyte condition; $33.2 \mathrm{ml} 25 \%$ magnesium sulfate, $200 \mathrm{ml}$ citrate per bag, $60 \mathrm{ml}$ calcium gluconate per group, can be adjusted appropriately base on the velocity of the replacement fluid and blood flow. The blood velocity is 150 to $180 \mathrm{ml} / \mathrm{min}$, the initial velocity of citric acid is 180 to $252 \mathrm{ml} / \mathrm{h}$, and the initial velocity of calcium gluconate is 25 to $35 \mathrm{ml} / \mathrm{h}^{[3]}$.

Patients in the control group were given routine nursing mode, in which nurses carried out health education to help patients understand medical safety knowledge, actively communicated with patients to answer their doubts, closely monitored patients' vital signs, and informed patients of relevant precautions. Patients in the research group received nursing safety management mode, as follows: (1) Set up a nursing safety management department, headed by an experienced and prestigious head nurse, made up of staff from the hemopurification center. The minister allocates and adjusts the work tasks, and makes the content of nursing work clearly planned. And department members discuss and develop targeted nursing programs, establish and improve the nursing safety management system, evaluate the nursing risks existing in the hemopurification center, and then adopt solutions according to the evaluation results. (2) Strengthen the safety management of the environment, for patients with different conditions, nursing staff should be personalized treatment, on the basis of the type of disease divided into special hemodialysis areas, and strictly restrict the entry and exit of irrelevant personnel. To grimly manage the sanitation of the hemopurification center, and conduct regular cleaning, disinfection, sterilization, for the critically ill patients and elderly patients in the sickbed to increase the guardrail, to avoid patients falling into bed. (3) To reinforce the safety management training of nursing staff, the minister conducts regular training and assessment for department members, so that nursing staff strengthen their nursing knowledge and practical operation ability, so as to provide better nursing services. (4) Promote the safety management of instruments. There are many kinds of instruments and equipment in the hemopurification center, so the nursing staff are required to distinguish the models and operation modes of each instrument, adjust the relevant indicators of each instrument appropriately, check and maintain the instrument regularly according to the standard, and disinfect the instrument after each use.

\subsection{Evaluation standard}

(1) The nursing quality scores of patients in the two groups after different nursing modes were compared. The nursing quality included service attitude, doctorpatient relationship, medical environment and professional skills. The total score of each item was 25 points, and the higher the score, the higher the nursing quality.

(2) The incidence of adverse events in the two groups after different nursing modes was compared, including catheter infection, equipment failure, bleeding during dialysis and falls. The number of cases occurred was counted, and the total incidence of adverse events was calculated.

(3) The nursing satisfaction of patients in the two 
groups after different nursing modes was compared. Questionnaire survey was adopted, and the contents of the questionnaire were very satisfied, relatively satisfied and dissatisfied. The number of the first two cases was the number of nursing satisfaction cases, and the proportion of the total number of cases was nursing satisfaction.

\subsection{Statistical calculation}

All data were substituted into SPSS 24.0 statistical software for calculation. $(\bar{x} \pm s)$ was used to represent the measurement data, and $\mathrm{t}$ was used to calculate; Represents the counting data as (\%), and calculates with $\chi^{2}$. If the final result $\mathrm{P}$ value is below 0.05 (excluding), it can be proved that the comparison difference is remarkable and statistically significant ${ }^{[4]}$.

\section{Results}

\subsection{Comparison of nursing quality between the two groups}

The service attitude, doctor-patient relationship, medical environment and professional skill scores of the research group were significantly higher than those of the control group $(P<0.05)$, and the detailed data are shown in Table 1.

Table 1. Comparison of nursing quality between the two groups $[(\bar{x} \pm s)$, score $]$

\begin{tabular}{cccccc}
\hline Group & $\boldsymbol{n}$ & Service attitude & Doctor-patient relationship & Medical environment & Professional skill \\
\hline Research group & 28 & $22.51 \pm 2.28$ & $23.21 \pm 1.55$ & $22.18 \pm 1.29$ & $23.14 \pm 1.38$ \\
Control group & 28 & $18.47 \pm 1.59$ & $16.71 \pm 2.56$ & $18.92 \pm 2.18$ & $17.30 \pm 2.21$ \\
$t$ & & 7.6908 & 11.4930 & 6.8100 & 11.8606 \\
$P$ & & 0.0000 & 0.0000 & 0.0000 & 0.00000 \\
\hline
\end{tabular}

2.2 Comparison of adverse events between the

was significantly lower than that in the control group two groups

$(P<0.05)$, and the detailed data were shown in Table 2 .

The incidence of adverse events in the research group

Table 2. Comparison of adverse events between the two groups [ $n(\%)]$

\begin{tabular}{ccccccc}
\hline Group & $\boldsymbol{n}$ & Catheter infection & Equipment failure & Hemodialysis bleeding & Fall & Total number of occurrence \\
\hline Research group & 28 & 0 & 0 & 1 & 0 & $1(3.57)$ \\
Control group & 28 & 2 & 3 & 4 & 2 & $11(39.29)$ \\
$\chi^{2}$ & & & & & 10.6061 \\
$P$ & & & & 0.0011 & 0.1 \\
\hline
\end{tabular}

\subsection{Comparison of nursing satisfaction between} the two groups

The satisfaction of patients in the research group was significantly higher than that in the control group $(P<0.05)$, and the detailed data were shown in Table 3.

Table 3. Comparison of nursing satisfaction between two groups of patients $[n(\%)]$

\begin{tabular}{cccccc}
\hline Group & $\boldsymbol{n}$ & Very satisfied & Relatively satisfied & dissatisfied & Total number of satisfaction \\
\hline Research group & 28 & 18 & 9 & 1 & $27(96.43)$ \\
Control group & 28 & 10 & 8 & 10 & $18(64.29)$ \\
$\chi^{2}$ & & & & 9.1636 \\
$P$ & & & & 0.0025 \\
\hline
\end{tabular}

\section{Discussion}

Hospital Hemopurification center can treat moderate and severe kidney diseases, the medical practices are closely related to the life safety of patients ${ }^{[5]}$. The continuous advance of the medical level has significantly improved the survival rate of patients with hemopurification, and the survival period has been significantly extended. Nevertheless, in practice, there are still many problems, and corresponding solutions need to be formulated for these problems, so as to increase the safety of patients and reduce the occurrence of nursery-patient disputes ${ }^{[6]}$. The main causes of these problems can be divided into system, environment, equipment, nursing staff, patients themselves. Among them, the imperfection of the system leads to the lack of effective constraints on the work of nursing staff, which further affects 
the carefulness of nursing work and the unclear responsibility and other problems; Unhygienic environment and equipment problems result in negative psychological state of patients, which seriously affects the treatment effect;Insufficient level of nursing staff will increase the incidence of medical errors; Patients themselves suffer a lot of pain due to various complications, at the same time, a longer treatment cycle will cause patients to produce negative emotions such as depression and anxiety, lose confidence in treatment, and seriously reduce the degree of cooperation, which has a huge impact on the nursing effect.

In this paper, research shows that, the team service attitude, the doctor-patient relationship, medical environment and the professional skill score were significantly higher than the control group $(P<0.05)$, the team the incidence of adverse events was obviously lower than the control group $(P<0.05)$, the team of patients to nursing satisfaction is significantly higher than the control group $(P<0.05)$, and fully manifests that the application of nursing safety management in hemopurification can effectively improve the quality of nursing, reduce the occurrence of adverse events, and enhance the patient's nursing satisfaction, the effect is obvious, and it is worth popularizing.

\section{References}

[1] Li YX. Nursing safety management countermeasures and implementation effect evaluation of hemopurification center [J]. Wisdom Health,2020,6(36):133-134.

[2] Shen J. Analysis and Countermeasure study on factors of nursing safety management in hemopurification center $[\mathrm{J}]$. Psychological Journal,2020,15(02):152.

[3] Ye AN, Zhu XQ, Zheng D, Zheng MH, Ruan JY. Problems in nursing management of hemopurification center and suggestions on standardized management $[\mathrm{J}]$. Journal of Traditional Chinese Medicine Administrati on,2019,27(07):70-71.

[4] Feng AY. Analysis and countermeasure of factors affecting safety management of hemopurification nursing $[\mathrm{J}]$. Journal of Traditional Chinese Medicine Management,2019,27(04):139-140.

[5] Han SJ. Preventive measures for the hidden danger of nursing management of dialysis patients in hemopurification center [J]. China Health Industry,2019,16(01):59-60.

[6] $\mathrm{Hu}$ K, Xu XM. Safety management factors and countermeasures of hemopurification nursing [J]. China Health Industry,2017,14(28):119-120. 